\title{
Prevention of Cervical Cancer
}

\author{
Yaa Achampong1, Fani Kokka ${ }^{2}$, Konstantinos Doufekas ${ }^{1}$, Adeola Olaitan ${ }^{{ }^{*}}$ \\ ${ }^{1}$ Department of Gynae-Oncology, University College Hospital London, London, UK \\ ${ }^{2}$ East Kent Gynae-Oncology Centre, Queen Elizabeth The Queen Mother Hospital, Kent, UK \\ Email: *Adeola.Olaitan@nhs.net
}

How to cite this paper: Achampong, Y., Kokka, F., Doufekas, K. and Olaitan, A. (2018) Prevention of Cervical Cancer. Journal of Cancer Therapy, 9, 79-88. https://doi.org/10.4236/jct.2018.91009

Received: November 12, 2017

Accepted: January 26, 2018

Published: January 29, 2018

Copyright $\odot 2018$ by authors and Scientific Research Publishing Inc. This work is licensed under the Creative Commons Attribution International License (CC BY 4.0).

http://creativecommons.org/licenses/by/4.0/ (c) (i) Open Access

\begin{abstract}
Cervical cancer is the fourth most common cancer in women and is responsible for 275,000 deaths worldwide each year. The burden of this disease lies in the developing world. However it is arguably the most preventable cancer. The Human Papilloma Virus (HPV) is responsible for almost all cases of Cervical Cancer. HPV is sexually transmitted and has a lifetime cumulative risk of infection of $80 \%$. National Cervical cytology screening programmes are used to detect cervical abnormalities, lesions and early cancers in as many eligible women as possible, saving lives and reducing morbidity. However uptake of screening is declining, and screening programmes have not been implemented in lower resource countries due to the cost and infrastructure required. Alternative screening methods have been implemented in such countries such as Visual Inspection with Acetic acid (VIA) but mortality and morbidity remain high. Since 2007, programmes of vaccination against the most oncogenic types of HPV have been rolled out internationally aiming to capture girls, and eventually potentially boys, before they become sexually active. These programmes have been largely successful, with good coverage and low adverse effects reported. Going forward, the vaccine and the advent of effective HPV screening and self testing mean that there needs to be a reorganisation of current cervical screening programmes.
\end{abstract}

\section{Keywords}

Cervical Cancer, HPV, Vaccine, Screening

\section{Introduction}

Cervical cancer is the fourth most common cancer in women worldwide, and presents an important global health issue [1].

It is a leading cause of death in women worldwide with 530,000 new cases and 275,000 deaths worldwide each year (WHO 2016), with the majority of cases 
occurring in less developed countries, especially in Sub-Saharan Africa. A fifth of all cases and over $25 \%$ of deaths occurred in India. Countries such as Kenya, Uganda and Nigeria rank high in the mortality stakes [2].

In Sub-Saharan Africa, 34.8 new cases of cervical cancer are diagnosed per 100,000 women annually and 22.5 per 100,000 women die from the disease. These figures compare with 6.6 and 2.5 per 100,000 women respectively in North America [3].

These differences are largely due to the lack of access to effective screening or services facilitating early detection and treatment. There is also a lack of accurate data due to a dearth of cancer registries in the worst affected countries and poor access to medical care resulting in fewer reported diagnoses.

\section{Methods}

\subsection{Epidemiology of HPV Infection}

Human papilloma virus is a key causative agent in the development of cervical cancer. The prevalence of HPV infection in cervical cancer is as high as $99 \%$.

HPV infection is sexually transmitted and often asymptomatic. Most sexually active men and women will be infected at some point in their lives. The lifetime cumulative risk of HPV infection is greater than $80 \%$. The peak time for acquiring the infection is shortly after becoming sexually active. Penetrative sex is not essential for transmission and skin to skin genital contact is well recognised as a mode of infection. Persistent infection is reported to occur in less than $10 \%$ and is defined as the presence of high risk HPV for longer than two years. Persistent infection with HPV increases the risk of cervical cancer [4].

In a national study from the US, 25\% of women between the ages of 14 and 19 years and $45 \%$ of women between the ages of 20 and 24 years were HPV positive [5]. HPV infection is more likely to persist in women over the age of 65 years, and a positive HPV test is therefore more likely to be clinically significant in this age group.

\subsection{HPV Subtypes}

There are 130 different HPV genotypes, subdivided into mucosal and cutaneous. Approximately 30 to $40 \mathrm{HPV}$ subtypes infect the genital mucosa and are categorised as low or high risk. Low risk types primarily cause benign ano-genital warts whereas high risk types are associated with ano-genital cancers.

The proportion of HPV infections that are high risk versus low risk varies with age. For example adolescents may be at similar risk for low and high risk infections whereas in women over the age of 30 years $50 \%-80 \%$ of HPV infections are with high risk subtypes.

HPV-16 and HPV-18 cause approximately 70\% of all invasive cervical cancers. HPV types $16,18,45,31,33,35,52$ and 58 together cause approximately $95 \%$ of cervical cancers. Most types of cervical cancer including squamous cancer, adeno-squamous cancer and adenocarcinoma are now thought to be associ- 
ated with HPV infections.

\subsection{HPV Prevalence}

In a national study from the US, $25 \%$ of women between the ages of 14 and 19 years and $45 \%$ of women between the ages of 20 and 24 years were HPV positive. A separate meta analysis of international prevalence found estimated crude and adjusted HPV prevalences (among women with normal cytological findings) worldwide of $7.2 \%$ and $11.7 \%$, respectively. The highest HPV prevalence was in Sub-Saharan Africa at $24.0 \%$, followed by Latin America and the Caribbean at 16.1\%, Eastern Europe at 14.2\%, and Southeastern Asia 14.0\%.

\subsection{Role of HPV in Malignant Transformation}

HPV infects the basal cells of squamous epithelium where keratinocytes undergo differentiation. In most cases the viral DNA stays separate from host DNA and forms an episome. In a subgroup of HPV infections the viral DNA integrates into host DNA, leading to malignant transformation. The viral E6 and E7 genes inhibit expression of host p53 and retinoblastoma tumour suppressor proteins. These proteins have an important role in cell cycle control and apoptosis and inactivation of their genes can induce malignant transformation.

Immunosuppression encourages persistent HPV infection. HIV co-infection can also promote HPV related malignant transformation at molecular level. Oral contraceptives and hormone replacement therapy (HRT) may up-regulate HPV expression [6]. Other risk factors that that may play a role in persistent HPV infection include active and passive smoking, host factors such as age and genetics and external factors, such as nutrition and environment.

\subsection{HPV Vaccination for the Prevention of Cervical Intra Epithelial Neoplasia}

Prophylactic vaccines against HPV entered national immunisation programs in many countries, including the UK. The development of national vaccination programs against high risk HPV is one of the most significant recent developments in cervical cancer prevention.

Vaccination generates HPV specific antibodies that bind to the virus and prevent cervical infection.

There are three types of HPV vaccines currently in use. Cervarix ${ }^{R}$ is a bivalent vaccine that protects against HPV types 16 and 18 (associated with cervical cancer). Gardasil ${ }^{\mathrm{R}}$ (also marketed as Silgard) is a quadri-valent vaccine. It protects against HPV types 6 and 11 (associated with ano-genital warts) as well as types 16,18 .

Gardasil 9 is a nine valent vaccine against HPV types 6, 11, 16, 18 and types $31,33,45,52,58$ (responsible for approximately 14\% of HPV associated cancers in women).

The US Food and Drug Administration (FDA) approved use of Gardasil in 
females aged 9 - 26 years in 2006 and use of Cervarix in 2009. The commercialisation of Cervarix and Gardasil represents a major milestone in the prevention of cervical cancer. In December 2014 the FDA also approved the 9-valent Gardasil 9.

Gardasil has been approved for vaccination programs in the USA, UK, Canada, Australia, New Very high levels of uptake have been reported in the UK, with $86 \%$ and $83 \%$ of eligible girls receiving the first and second doses of the HPV vaccine respectively. This high level of uptake results from the fact that the vaccine is delivered through schools [7].

The United States, Canada and Australia also offer vaccination to adolescent boys to prevent against anal and oropharyngeal cancers. HPV vaccination is not currently offered to boys or men in the UK.

Comparing HPV immunisation programs between countries is difficult because the delivery systems often differ significantly [8]. Recent evidence from the UK on the performance of cytology and colposcopy in vaccinated women suggests that in younger women who have received the vaccine there is a significant reduction in low and high grade cytology and histology compared with unvaccinated women [9]. This may reduce the workload of the colposcopy clinics.

HPV vaccination will be most beneficial in resource poor countries that lack organised screening programs and where the burden of cervical cancer is highest.

Table 1 illustrates countries with a national vaccination programme.

Australia was the first country to roll out a formal national school based HPV vaccination programme. The United States, Canada and Australia also offer vaccination to adolescent boys to prevent against anal and oropharyngeal cancers. HPV vaccination is not currently offered to boys or men in the UK.

Comparing HPV immunisation programs between countries is difficult because the delivery systems often differ significantly. The UK is the only European country that has a national school based program. Like the UK, Canada and Australia's programs are school based although different provinces target different cohorts of girls between the ages of 9 and 17 years. School based vaccination programs generally achieve higher coverage than on demand systems where it relies on the patient to request the vaccine. Some countries offer vaccination up to the age of 26 years. The effects of vaccination in women of older age have not been sufficiently studied.

In terms of coverage, it is estimated that 118 million women across 64 countries have received at least one dose of the HPV vaccine, with 47 million receiving all 3 doses. However only $1.1 \%$ of these women were in low resource countries [10].

\subsection{Evidence of Efficacy}

Large international randomised trials have evaluated both Cervarix and Gardasil and have shown that both vaccines are over 99\% effective in preventing 
Table 1. National HPV vaccination programmes.

\begin{tabular}{|c|c|}
\hline Country & Year National Vaccination Programme Commenced \\
\hline Austria & 2006 \\
\hline Belgium & 2007 \\
\hline Croatia & 2016 \\
\hline Denmark & 2009 \\
\hline Finland & 2013 \\
\hline France & 2007 \\
\hline Germany & 2007 \\
\hline Greece & 2007 \\
\hline Hungary & 2014 \\
\hline Iceland & 2011 \\
\hline Ireland & 2008 \\
\hline Italy & 2007 \\
\hline Latvia & 2009 \\
\hline Luxembourg & 2008 \\
\hline Macedonia & 2009 \\
\hline Netherlands & 2009 \\
\hline Norway & 2009 \\
\hline Portugal & 2007 \\
\hline Romania & 2008 \\
\hline Slovenia & 2009 \\
\hline Spain & 2007 \\
\hline Sweden & 2010 \\
\hline Switzerland & 2008 \\
\hline UK & 2008 \\
\hline US & 2008 \\
\hline Australia & 2007 \\
\hline Canada & 2006 \\
\hline Ghana & 2013 \\
\hline Niger & 2013 \\
\hline Sierra Leone & 2013 \\
\hline Rwanda & 2014 \\
\hline
\end{tabular}

precancerous lesions associated with HPV-16 and 18 in young women who are HPV naïve [11] [12] [13].

Longer term studies are however needed to ascertain if booster doses are required.

The major trials have used antibody titres or prevention of CIN2 and CIN3 as 
primary end points which are surrogate markers for the protection against cervical cancer. Evidence on prevention of cervical cancer is not available.

Current vaccines may offer some degree of cross protection against other HPV types; however this effect is probably modest.

A study comparing genoprevalance of all HPV types in Australian women aged 18 - 24 before the introduction of the national vaccination programme in 2007 (2005-2007) and after (2010-2011), found a significant drop in HPV prevalence from $28.7 \%$ to $6.7 \%$ ( $\mathrm{p}<0.001)$ in the post vaccine group [14].

There are still unanswered questions about the long term cost effectiveness and safety of current HPV vaccines. In the UK there has also been criticism of the decision to limit vaccination to adolescent girls.

\subsection{Safety of HPV Vaccination}

In general all three HPV vaccines appear to be safe and well tolerated. They pose no infection or oncogenic risk. The World Health Organization Global Advisory Committee for Vaccine Safety (GACVS) concluded that potential benefits outweigh any harms.

Commonly reported adverse effects include pain, swelling and redness at the injection site, nausea, headache, fever, musculoskeletal pain and syncope. Cervarix is recognized to be a more painful vaccination. The anaphylaxis rate for Gardasil has been reported as 2.6 per 100,000 doses which is higher than for other vaccines albeit still rare. An anaphylactic reaction is a contraindication for a subsequent dose.

There have been no deaths attributable to the HPV vaccines up to date.

The HPV vaccine is not recommended for use in pregnancy. Where it has been inadvertently administered during pregnancy no adverse pregnancy outcomes of fetal malformations have been reported [15].

\subsection{The Future of HPV Vaccination}

As up to $30 \%$ of cervical cancers are caused by HPV subtypes not covered by the vaccine, the need for routine screening will remain.

HPV vaccines can potentially reduce the burden of cervical cancer on women and health services. Significant challenges remain in achieving a greater coverage of adolescents and in reducing the cost of HPV vaccines, thus making them more accessible to countries in the developing world, where they are most needed [16].

Progress is being made in developing novel therapeutic HPV vaccines to treat existing HPV infections and diseases. Therapeutic vaccines aim to generate cell mediated immunity and may provide an option for treating HPV associated disease [17] [18].

\subsection{Screening and HPV Testing}

Cervical cancer screening is a way to detect abnormal cervical cells, precancerous cervical lesions and early cervical cancers. Both precancerous lesions and 
early cervical cancer can be treated very successfully.

The introduction of cervical cytology screening has been particularly effective in preventing advanced disease, saving hundreds of thousands of lives. Screening is estimated to have reduced the incidence of cervical cancer by up to $80 \%$ [19].

Cervical screening coverage rates vary across western countries and have declined over the past 8 years, particularly in young women. In the UK, national screening program coverage rates have been around $80 \%$. Following a surge in screening in 2007-8 after the diagnosis and subsequent death of a celebrity from Cervical cancer, rates have dropped back to pre 2008 levels (Cancer Research UK). In Italy coverage rates have never been above 50\% in young women [20].

There are initiatives in the UK to try increase coverage including educating women on the number of lives saved annually through screening as well as the death rate for cervical cancer, written invitations and reminders of missed appointments instead of phone calls from nurses and admin staff and increasing access and availability, offering screening at GUM clinics and by trained practice Nurses for example [21].

Cervical cytology screening programs are resource heavy and thus uptake is even lower in median to low income countries. A study investigating the uptake of cervical cytology screening across the globe showed huge variation. They examined 57 countries and Pap smear uptake averaged 45\% in developed and 19\% in developing countries with uptake as high as $80 \%$ in Luxembourg and as low $1 \%$ in Bangladesh [22].

Visual inspection with acetic acid (VIA) or Lugol's iodine (VILI) is a simple and cheap screening method used in low income countries. VIA can be performed at any time during the menstrual cycle, immediately postpartum or after pregnancy termination without need for an expensive laboratory set up, facilitating opportunistic testing. There is less reliance on sequential testing, therefore empowering the examining clinician 'to see and treat' any lesions or area of abnormality.

\section{The Future of Cervical Screening}

As HPV related precancerous lesions become increasingly rare in countries where HPV vaccines are available there may be a need for major reorganisation of cervical cancer prevention guidelines.

An important objective is to define evidence based screening strategies for girls vaccinated against HPV. Tailored screening protocols based on vaccination status, rather than "a one size fits all approach" are needed until a herd immunity effect is achieved.

A consensus conference that took place in Italy in 2015 [23] recommended that vaccinated women should start screening at the age of 30 years with HPV test. There is a strong rationale for applying longer intervals for rescreening HPV negative women although more research is needed to define an optimal interval. Research published in November 2017 by Cancer Research UK [24] 
used a microsimulation model using real published data to determine appropriate screening intervals for vaccinated women, and suggested that in the UK, women who received the bivalent vaccine may only need three cervical screens in their life time. Longer screening intervals and delayed onset of screening can have a positive economic and organisational impact by reducing workload and unnecessary referrals and treatments.

\section{Cervical Cancer Prevention in Low Resource Settings}

The development of molecular testing for HPV has advanced the opportunity for self testing, which may be of high value in low resource countries or countries with low screening uptake. HPV self-testing can overcome the potential obstacles of access to trained health care professionals as well as cultural variations in attitude towards intimate examinations.

Self-sampling for HPV detection shows high concordance (96.8\%) with physician taking sampling [25].

HPV testing alone appears to be more sensitive compared with cytology. The sensitivity of HPV testing for the detection of CIN2 and CIN3 has been reported as $94.6 \%$ compared to $55.4 \%$ for Pap testing [26]. Its negative predictive value is high.

It is however, less specific and has a lower positive predictive value compared with cytology. Despite this, HPV testing may be preferred in countries where restricted infrastructure reduces the effectiveness of cytology screening programs. In addition, as women in low resource settings will be screened only a few times in their lives the high sensitivity of HPV testing is of paramount importance [27].

Other biomarkers may be used after HPV testing in the future to improve the specificity of screening, which may reduce the number of women being unnecessarily referred to secondary care for colposcopy [18].

As with all diseases, education about avoiding risks such as smoking, early coitarche, unprotected intercourse with multiple partners and increased surveillance in women with HIV is the key.

\section{References}

[1] WHO (2016) Human Papilloma Virus (HPV) and Cervical Cancer. WHO Fact Sheet. http://www.who.int/mediacentre/factsheets/fs380/en/

[2] Bray, F., et al. (2013) Global Estimates of Cancer Prevalence for 27 Sites in the Adult Population in 2008. International Journal of Cancer, 132, 1133-1145. https://doi.org/10.1002/ijc.27711

[3] Ferlay, J., et al. (2013) GLOBOCAN 2012 v1.0, Cancer Incidence and Mortality Worldwide: IARC CancerBase No. 11 [Internet]. International Agency for Research on Cancer, Lyon. http://globocan.iarc.fr

[4] Goodman, A. (2015) HPV Testing as a Screen for Cervical Cancer. BMJ, 350, h2372. https://doi.org/10.1136/bmj.h2372

[5] Kahn, J. (2009) HPV Vaccination for the Prevention of Cervical Intraepithelial 
Neoplasia. The New England Journal of Medicine, 361, 271-278. https://doi.org/10.1056/NEJMct0806938

[6] Smith, E. and Turek, L. (2003) Prevalence and Persistence of Human Papilloma Virus in Postmenopausal Women. Cancer Detection and Prevention, 27, 472-480. https://doi.org/10.1016/S0361-090X(03)00104-1

[7] Quah, Y.L. and Aggarwal, I.M. (2017) 10 Minute Consultation. Discussing Human Papilloma Virus Vaccination. BMJ, 357, j2730. https://doi.org/10.1136/bmj.j2730

[8] Crosbie, E.J. and Brabin, L. (2010) Cervical Cancer: Problem Solved? Vaccinating Girls against Human Papillomavirus. BJOG, 117, 137-142. https://doi.org/10.1111/j.1471-0528.2009.02369.x

[9] British Society for Colposcopy and Cervical Pathology (2013) The Implications of HPV Vaccinations on Coploscopy.

http://www.bsccp.org.uk/healthcare-professionals/professional-news/the-implicatio ns-of-hpv-vaccinations-for-colposcopy/

[10] Bruni, L. and Diaz, M. (2016) Global Estimates of Human Papillomavirus Vaccination Coverage by Region and Income Level: A Pooled Analysis. The Lancet Global Health, 4, e453-e463. https://doi.org/10.1016/S2214-109X(16)30099-7

[11] Garland, S.M., et al. (2007) Females United to Unilaterally Reduce Endo/Ectocervical Disease (FUTURE) Investigators. Quadrivalent Vaccine against Human Papilloma Virus to Prevent Anogenital Diseases. The New England Journal of Medicine, 357, 1928-1943. https://doi.org/10.1056/NEJMoa061760

[12] FUTURE II Study Group (2007) Quadrivalent Vaccine against Human Papilloma Virus to Prevent High-Grade Cervical Lesions. The New England Journal of Medicine, 357, 1915-1927

[13] Centers for Disease Control and Prevention (2015) Human Papilloma Virus (HPV) Vaccine Safety. http://www.cdc.gov/vaccinesafety/vaccines/hpv-vaccine.html

[14] Tabrizi, S. and Brotherton, J.M.L. (2012) Fall in Human Papillomavirus Prevalence Following a National Vaccination Program. The Journal of Infectious Diseases, 206, 1645-1651. https://doi.org/10.1093/infdis/jis590

[15] Narducci, A., Einarson, A. and Bozzo, P. (2012) Human Papilloma Virus Vaccine and Pregnancy. Canadian Family Physician, 58, 268-269.

[16] Ma, B., Roden, R. and Wu, T.C. (2010) Current Status of HPV Vaccines. Journal of the Formosan Medical Association, 109, 481-483. https://doi.org/10.1016/S0929-6646(10)60081-2

[17] Trimble, C.L. and Frazer, I.H. (2009) Development of Therapeutic HPV Vaccines. The Lancet Oncology, 10, 975-980. https://doi.org/10.1016/S1470-2045(09)70227-X

[18] Yang, A., et al. (2016) Perspectives for Therapeutic HPV Vaccine Development. Journal of Biomedical Science, 23, 75. https://doi.org/10.1186/s12929-016-0293-9

[19] Sasieni, P., Castanon, A. and Cuzick, J. (2009) Effectiveness of Cervical Screening with Age: Population Based Case-Control Study of Prospectively Recorded Data. $B M J, 339, \mathrm{~b} 2968$.

[20] Martin-Hirsch, P. (2013) Commentary on "Use of a High-Risk Human Papilloma Virus DNA Test as the Primary Test in a Cervical Cancer Screening Programme: A Population-Based Cohort Study". BJOG, 120, 1267-1268.

[21] Cancer Research UK (2016) Cervical Cancer Statistics: Prevention. http://www.cancerresearchuk.org/health-professional/cancer-statistics/statistics-bycancer-type/cervical-cancer/diagnosis-and-treatment

[22] Gakidou, E., Nordhagen, S. and Obermeyer, Z. (2008) Coverage of Cervical Screen- 
ing in 57 Countries: Low Average Levels and Large Inequalities. PLOS Medicine, 5, e132. https://doi.org/10.1371/journal.pmed.0050132

[23] Rossi, G., et al. (2017) Cervical Cancer Screening in Women Vaccinated against Human Papilloma Virus Infection: Recommendations from a Consensus Conference. Preventive Medicine, 98, 21-30. https://doi.org/10.1016/j.ypmed.2016.11.020

[24] Landy, R., Windridge, P., et al. (2017) What Cervical Screening Is Appropriate for Women Who Have Been Vaccinated against High Risk HPV? A Simulation Study. International Journal of Cancer, 142, 709-718.

[25] Ketelaars, P.J.W., et al. (2017) High Risk Human Papilloma Virus Detection in Self-Sampling Compared to Physician Taken Smear in a Responder Population of the Dutch Cervical Screening: Results of the VERA Study. Preventive Medicine, $101,96-101$.

[26] Mayrand, M.H., et al. (2007) Human Papilloma Virus DNA versus Papanicolaou Screening Tests for Cervical Cancer. The New England Journal of Medicine, 357, 1579-1588. https://doi.org/10.1056/NEJMoa071430

[27] Lazcano Ponce, E., et al. (2011) Self-Collection of Vaginal Specimens for Human Papilloma Virus Testing in Cervical Cancer Prevention (MARCH): A Community-Based Randomized Controlled Trial. The Lancet, 378, 1868-1873.

https://doi.org/10.1016/S0140-6736(11)61522-5 